\title{
Real-time interactive streams and temporal objects language
}

\author{
Mauricio Toro, Myriam Desainte-Catherine, David Janin, Yann Orlarey
}

October 4, 2018

\begin{abstract}
We introduce a real-time interactive streams language based on the model of temporal objects in order to (1) study various typing features for timed streams and (2) define a hierarchical calculus for stream processus. The present document deals only with formalisation, and introduces the typing features.
\end{abstract}

In the sequel, we consider a discrete notion of time where time values are taken in $\mathbb{N}$. At the abstract level this is just enough. At a more concrete level, these time values can either be interpreted as samples numbers, or frame numbers, or whatever else...

\section{Basic stream features}

A stream is a partial map $S: \mathbb{N} \rightarrow \mathbb{R}^{n}$. The domain of such a stream $S$, written $\operatorname{dom}(S)$ is assumed to be a segment of $\mathbb{N}$ of the form

$$
\operatorname{dom}(S)=[s ; s+d[=\{m \in \mathbb{N}: s \leq m<s+d\}
$$

where $\operatorname{start}(S)=s \in \mathbb{N}$ is the starting delay of stream $S$, duration $(S)=d \in$ $\overline{\mathbb{N}}$ is the duration of stream $S$ - with $\overline{\mathbb{N}}=\mathbb{N}+\{\infty\}-$ and $\operatorname{dim}(S)=n \in \mathbb{N}$ is dimension of stream $S$.

Such a (finite) stream, with an implicit time line going from left to right, can be drawn as follows:

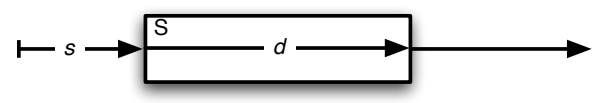

As far as semantic is concerned, we assume that any stream belongs to a program that has been fired at time 0 . We must also keep in mind that when a stream is undefined, this does not mean that the stream programs cannot handle it. 
More precisely, when $t \notin \operatorname{dom}(S)$, we write $S(t)=\perp$ to denote the fact that $S(t)$ is undefined. But, this undefined value can be used in computation, as illustrated, for instance, by the sequential composition below.

The somehow orthogonal problematic raised by computational availability or knowledge is nevertheless of a crucial importance especially in interactive manipulation of streams where some streams can be external inputs. It is thus discussed in Section 3.

For every $x \in \mathbb{R}$, and $n \in \mathbb{N}$, we shall write $x^{n}$ to denote, with a little abuse of notation, the vector $x^{n}=(x, x, x, \cdots, x)$ of length $n$.

For the sake of completeness of the stream algebra, we allow stream $S$ of dimension 0 hence $x^{0}$ is also defined as a special zero-length vector written $\epsilon$.

A zero-dimensional stream is thus a mapping from $\operatorname{dom}(S) \rightarrow \mathbb{R}^{0}$ where $\mathbb{R}^{0}=\{\epsilon\}$. It is thus completely determined by its domain.

In the sequel, for every $u \in \mathbb{R}^{m}$ and $v \in \mathbb{R}^{n}$ we shall write $u v \in \mathbb{R}^{m+n}$ for the vector obtained from $u$ and $v$ just by concatenating its values. With such a notation, we have, for every $u \in \mathbb{R}^{m}, \epsilon u=u \epsilon=u$ hence this concatenation of vectors of floats really just behaves like concatenation of finite words.

The set of streams is denoted by $\mathcal{S}$.

\section{Operators on streams}

Let $S_{1}$ and $S_{2}$ be two streams with

$$
\operatorname{dom}\left(S_{1}\right)=\left[s_{1}, s_{1}+d_{1}\left[\text { and } \operatorname{dim}\left(S_{1}\right)=n_{1}\right.\right.
$$

and

$$
\operatorname{dom}\left(S_{2}\right)=\left[s_{2}, s_{2}+d_{2}\left[\text { and } \operatorname{dim}\left(S_{2}\right)=n_{2}\right.\right.
$$

\subsection{Primitive operators}

Sequential composition. The sequential composition $S_{1} \cdot S_{2}$ of streams $S_{1}$ and $S_{2}$ with $\operatorname{dim}\left(S_{1}\right)=\operatorname{dim}\left(S_{2}\right)=n$ and duration $\left(S_{1}\right)<+\infty$ is defined by $\operatorname{dom}\left(S_{1} \cdot S_{2}\right)=\left[s_{1}, s_{1}+s_{2}+d_{1}+d_{2}\left[{ }^{1}, \operatorname{dim}\left(S_{1} \cdot S_{2}\right)=n\right.\right.$ with, for every $x \in \operatorname{dom}\left(S_{1} \cdot S_{2}\right)$,

$$
S_{1} \cdot S_{2}(x)= \begin{cases}S_{1}(x) & \text { if } s_{1} \leq x<s_{1}+d_{1} \\ 0^{n} & \text { if } 0 \leq x-\left(s_{1}+d_{1}\right)<s_{2}, \\ S_{2}\left(x-\left(s_{1}+d_{1}\right)\right) & \text { if } s_{2} \leq x-\left(s_{1}+d_{1}\right)<s_{2}+d_{2}\end{cases}
$$

The result of a stream sequential product of stream $S_{1}$ with stream $S_{2}$ can be illustrated as follows:

\footnotetext{
${ }^{1}$ with, for every $m \in \mathbb{N}+\infty, m+\infty=\infty+m=\infty$
} 


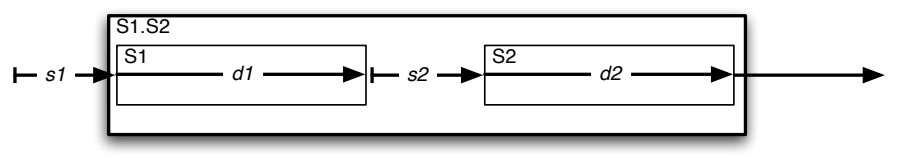

Parallel composition. The parallel composition $S_{1} \| S_{2}$ of streams $S_{1}$ and $S_{2}$ is defined by $\operatorname{dom}\left(S_{1} \| S_{2}\right)=\left[\min \left(s_{1}, s_{2}\right), \max \left(s_{1}+d_{1}, s_{2}+d_{2}\right)-\right.$ $\min \left(s_{1}, s_{2}\right)$ [, i.e. $\operatorname{dom}\left(S_{1} \| S_{2}\right)$ is the least segment that contains $\operatorname{dom}\left(S_{1}\right) \cup$ $\operatorname{dom}\left(S_{2}\right)$, with $\operatorname{dim}\left(S_{1} \| S_{2}\right)=n_{1}+n_{2}$. For every $x \in \operatorname{dom}\left(S_{1} \| S_{2}\right)$,

$$
\left(S_{1} \| S_{2}\right)(x)= \begin{cases}S_{1}(x) S_{2}(x) & \text { if } x \in \operatorname{dom}\left(S_{1}\right) \cap \operatorname{dom}\left(S_{2}\right), \\ S_{1}(x) 0^{n_{2}} & \text { if } x \in \operatorname{dom}\left(S_{1}\right)-\operatorname{dom}\left(S_{2}\right), \\ 0^{n_{1}} S_{2}(x) & \text { if } x \in \operatorname{dom}\left(S_{2}\right)-\operatorname{dom}\left(S_{1}\right) .\end{cases}
$$

The result of the parallel product of $S_{1}$ and $S_{2}$ as above with $s_{1}<s_{2}$ and $s_{2}+d_{2}<s_{1}+d_{1}$ can be isslutrated as follows:

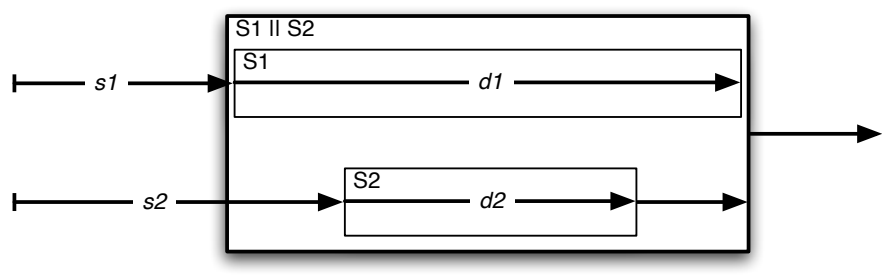

Synchronized parallel composition. The synchronized parallel composition $S_{1} \mid S_{2}$ of streams $S_{1}$ and $S_{2}$ is defined by $\operatorname{dom}\left(S_{1} \mid S_{2}\right)=\left[s_{1}, \max \left(s_{1}+\right.\right.$ $\left.d_{1}, s_{2}+d_{2}\right)-s_{1}$ [, i.e. $\operatorname{dom}\left(S_{1} \mid S_{2}\right)$ is the least segment that contains $\operatorname{dom}\left(S_{1}\right) \cup$ $\operatorname{dom}\left(S_{2}\right)$, with $\operatorname{dim}\left(S_{1} \mid S_{2}\right)=n_{1}+n_{2}$, such that $S_{2}$ is retarded or avanced to start at the same time than the $S_{1}$. Let $S_{2}^{\prime}$ such that $\operatorname{start}\left(S_{2}\right)=s_{1}$, duration $\left(S_{2}^{\prime}\right)=d_{2}$, be the stream resulting when retarding or avancing $S_{2}$ to start at the same time than $S_{1}$.

$$
\left(S_{1} \mid S_{2}\right)=S_{1} \| S_{2}^{\prime}
$$

The result of the parallel product of $S_{1}$ and $S_{2}$ as above with $s_{1}<s_{2}$ and $s_{2}+d_{2}<s_{1}+d_{1}$ can be isslutrated as follows: 

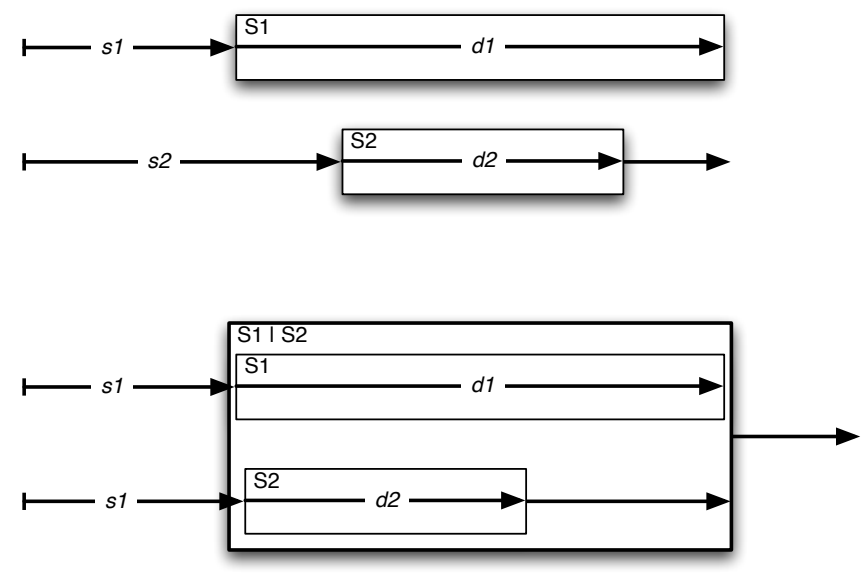

Restriction. The restriction composition $S_{1} / S_{2}$ of streams $S_{1}$ and $S_{2}$ is defined essentially in the same way $S_{1} \| S_{2}$ is defined but with a reduced domain $\operatorname{dom}\left(S_{1} / S_{2}\right)=\operatorname{dom}\left(S_{2}\right)$.

In particular, when $S_{2}$ is a zero-dimensional stream, the restriction stream $S_{1} / S_{2}$ is essentially as stream $S_{1}$ restricted to domain $\operatorname{dom}\left(S_{2}\right)$.

The result of a the restriction product of $S_{1}$ and $S_{2}$ with the same hypothesis as above can be illustrated as follows:

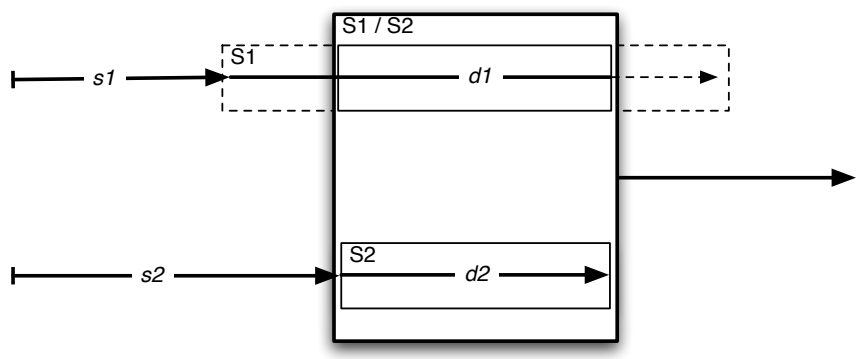

\subsection{Streams operators}

Transformation. Given a stream transformation $F$ and a given stream $S$ we want to define $F(S)$.

Given $s=\operatorname{start}(S)$ and $d=\operatorname{duration}(S)$ we assume that $\operatorname{start}(F(S)=$ $s+\delta_{F}(d)$, i.e. the extra start delay $\delta_{F}(d)$ of $F(S)$ compared to $S$ only depends on the duration $d$ of $S$, and that duration $(S)=d . \alpha_{F}(d)$, i.e. the duration of $F(S)$ is a factor of the duration of $S$ with coeficient factor $\alpha_{F}(d)$ that also only depends on $d$.

When $\delta_{F}(d)=0$ and $\alpha_{F}(d)=1$, the result of the application of a stream transformation can be illustrated as follows: 


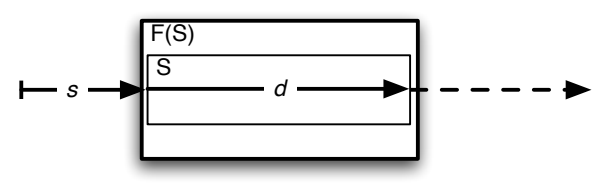

Reverse. Reverse corresponds to audio reverse. Let us consider the two following reverse operators : reverse $(S)$ computes the reverse stream by accessing the samples of $S$ from left to right. Reserse does not change the duration nor the start of the original stream.

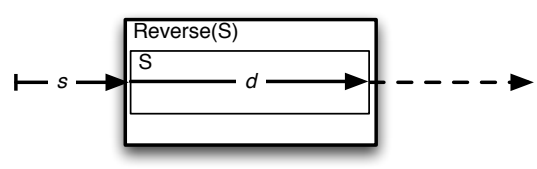

Stream syntax vs stream semantics. In the remainder of the text, we use the same notation for both stream expressions and stream themselves. This is done on purpose in order to keep some reasonably simple notations.

Whether a given streams expression is interpreted as itself or as the stream it denotes shall always remain clear from the context or will be explicitly mentioned.

The set of streams expressions is denoted by $\mathcal{E}$.

\subsection{Derived operators}

For all $n \in \mathbb{N}, s \in \mathbb{N}$ and $d \in \overline{\mathbb{N}}$, let $0_{s, d}^{n}$ denote the stream with domain $\left[s, s+d\left[\right.\right.$ and with constant value $0^{n}$.

Delays. The first delay operator one can define on a stream $S$ with $\operatorname{dom}(S)=$ $[s, s+d[$ and $\operatorname{dim}(S)=n$ by some constant $\delta$ is given by

$$
\operatorname{delay}(S, \delta)=0_{\delta, 0}^{n} \cdot S
$$

with $\operatorname{dom}\left(0_{\delta, 0}^{n} . S\right)=[s+\delta, s+d+\delta[$.

Extract operator. This operator extracts a finite segment of a (finite or infinite) stream.

$$
\operatorname{extract}(S, s, d)=S / T
$$

with $T=0_{s, s+d}^{0}$ a zero-dimensional stream with domain $[s, s+d[$.

Sum operator. This operator merges two streams $S, R$. Sum is a particular case of $F(S \| R)$ in which $F$ takes a bidimensional stream, $S: \mathbb{N} \rightarrow \mathbb{R}^{2}$ and returns a unidimensional stream, $S: \mathbb{N} \rightarrow$ mathbbR. In the case of sum, $\delta_{F}(d)=0$ and $\alpha_{F}(d)=1$. 


\section{Bandpass filter.}

\section{Temporal properties}

In this section, we want to investigate different temporal properties of streams like computational availability and computational necessity of streams. These notions appear as soon as we handle various type of streams : from input streams that are known sequentially in real time to file streams that are known at any time.

\subsection{Input and buffered streams}

Let us first review the characteristic of input or buffered streams.

Input stream An input stream is a stream denoted by an expression of the form $I_{p}$ where $p$ is an input port. For such a stream, we necessarily have $\operatorname{dom}\left(I_{p}\right)=[0, \infty[$. At any time $t$, provided the input stream is buffered, $S\left(t^{\prime}\right)$ is known only for $t^{\prime} \in[0, t]$.

Buffered stream A file stream is a stream denoted by an expression of the form $B_{f}$ where $f$ is a file descriptor. For such a stream, we necessarily have $\operatorname{dom}(B)=\left[s, d\left[\right.\right.$ with $d<\infty$. At any time $t, S\left(t^{\prime}\right)$ is known (though possible with undefined value) for arbitrary $t^{\prime} \in \mathbb{N}$.

\subsection{Computational availability and knowledge}

As already mentioned, handling streams in real time context poses problems of computational availability of a stream $S$ : at a given time $t$, for which $t^{\prime}$ the value $S\left(t^{\prime}\right)$ is available for computation.

Implicitly, we assume here that streams are buffered as much as needed. The minimal size of these buffer is handled by the orthogonal notion of computational necessity presented below.

We have two diferent definitions : computational availability and computational knowledge of streams. Let $\mathrm{S}$ be a stream :

- $\operatorname{avail}(S, t)$ is the set of index of samples of $\mathrm{S}$ that are available at execution time t.

- $k(S, t)$ is the interval containing the dates of execution at which $\mathrm{S}(\mathrm{t})$ is known. For example, if we assume that we bufferize until the end of execution all the streams that are handled by the program, we can state that $k(I, t)=[t, \infty[$ with $I$ denoting an input stream (in realtime). 
Note that there is a relation between avail and $k$. In a future version of this writting, only one should remain (the simplest one to express all the operators).

$$
\operatorname{avail}(s, t)=\left\{t^{\prime} \mid t \in k\left(s, t^{\prime}\right)\right\} k(s, t)=\left\{t^{\prime} \mid t^{\prime} \in \operatorname{avail}(s, t)\right\}
$$

We also want to define the notion of computational necessity. We thus define the computational necessity of a stream $S$ used in a program $F(S)$. We propose the following definition : at a given time $t$ during execution, the computational necessity is the set of dates $t^{\prime}$ such that the values of $S\left(t^{\prime}\right)$ must be known to compute $(F(S))(t)$.

The notion of stream availability goes in pair with the somehow dual notion of computational necessity of a stream $S$ used in a program $F(S)$ : at a given time $t$, for which time $t^{\prime}$ the values of $S\left(t^{\prime}\right)$ must be known to compute $(F(S))(t)$.

\section{Computational Availability}

\subsection{Definition}

Formally, given $\mathcal{E}$ the class of stream expressions built from elementary streams either constant or of the form $I_{p}$ or $B_{f}$ combined by the stream operators defined above, we define a mapping

$$
\text { avail }: \mathcal{E} \times \mathbb{N} \rightarrow \mathcal{P}(\mathbb{N})
$$

that maps any stream expression $S$ and time value $t \in \mathbb{N}$ to a set avail $(S, t)$ such that for every $t^{\prime} \in \operatorname{avail}(S, t), S\left(t^{\prime}\right)$ is computable (be it defined or undefined).

In the following for any stream $S$, let us denote by $e(S)$ the date of the last sample of $S$, that is $s(S)+d(S)$.

\subsection{Availability of constant streams}

For an external input stream $I_{p}$ we have

$$
\begin{gathered}
\operatorname{avail}\left(I_{p}, t\right)=[0, t] \\
k\left(I_{p}, t\right)=[t, \infty[
\end{gathered}
$$

since $s\left(I_{p}\right)=0$ and $d\left(I_{p}\right)=\infty$. Of course, this implies that the entire input stream from time 0 to time $t$ has been buffered.

By contrast, for any file stream $B_{f}$, we have

$$
\begin{gathered}
\operatorname{avail}\left(B_{f}, t\right)=[0, \infty[ \\
k\left(B_{f}, t\right)=[0, \infty[
\end{gathered}
$$

Of course, this also implicitly implies that the file associated to the stream $B_{f}$ is kept available until the end of execution. 


\subsection{Sequential composition}

Let $S_{1}$ and $S_{2} \in \mathcal{E}$ be two arbitrary stream expressions.

$$
\operatorname{avail}\left(S_{1} \cdot S_{2}, t\right)=\operatorname{avail}\left(S_{1}, t\right) \cup \operatorname{shift}\left(\operatorname{avail}\left(S_{2}, t\right), s\left(S_{1}\right)+d\left(S_{1}\right)\right)
$$

with, for all $X \subseteq \mathbb{N}$, all $d \in \mathbb{N}$,

$$
\begin{gathered}
\operatorname{shift}(X, d)=\{n+d: n \in X\} \\
k\left(S_{1} . S_{2}, t\right)=\left[t, e\left(S_{1}\right)\left[\cup \operatorname{shift}\left(k\left(S_{2}, t\right), s\left(S_{1}\right)+d\left(S_{1}\right)\right)\right.\right.
\end{gathered}
$$

\subsection{Parallel composition}

Let then $S_{1}$ and $S_{2} \in \mathcal{E}$ be two arbitrary stream expressions.

$$
\operatorname{avail}\left(S_{1} \| S_{2}, t\right)=\operatorname{avail}\left(S_{1}, t\right) \cap \operatorname{avail}\left(S_{2}, t\right)
$$

Indeed, at time $t$, the ??

\subsection{Delay operator}

Let then $S \in \mathcal{E}$ be an arbitrary stream.

$$
\begin{gathered}
\operatorname{avail}(\operatorname{delay}(S, \delta), t)=\operatorname{shift}(\operatorname{avail}(S, t), \delta) \\
k(S, t)=[t+\delta, \infty[
\end{gathered}
$$

\subsection{Restriction operator}

Let then $S_{1}$ and $S_{2} \in \mathcal{E}$ be two arbitrary stream expressions.

$$
\operatorname{avail}\left(S_{1} / S_{2}, t\right)=\operatorname{avail}\left(S_{2}, t\right)
$$

\subsection{Transformation operator}

Let then $S \in \mathcal{E}$ be an arbitrary stream.

$$
\operatorname{avail}(F(S), t)=\operatorname{shift}\left(\operatorname{avail}(S, t), \delta_{F}(d)\right) \cup \operatorname{shift}\left(\left[d, d . \alpha_{F}(d)\left[, \delta_{F}(d)\right)\right.\right.
$$

\subsection{Reverse operator}

Availibility of the result of the application of reverse is defined as follows :

$$
\operatorname{avail}(\operatorname{reverse}(S), t)=[e(S)-t, e(S)]
$$




\section{Computational necessity}

\subsection{Definition}

Given $\mathcal{E}$ the class of stream expressions,

$$
\text { req }: \mathcal{E} \times \mathcal{E} \times \mathbb{N} \rightarrow \mathcal{P}(\mathbb{N})
$$

that maps any stream expression $S$ possibly used in a (more complex) stream expression $E$ and any time value $t \in \mathbb{N}$ to an interval

$$
\operatorname{req}(S, E, t)=[t-\operatorname{pr}(S, E, t), t+f r(S, E, t)]
$$

defined as the least interval such that, for every $t^{\prime} \in \operatorname{avail}(E, t), E(t)$ is effectively computable when we assume (or force) the availability of signal $S$ at time $t$ to be restricted to $\operatorname{req}(S, E, t)$.

In this definition, we aim at ensuring that for every substream expression $S$ in $E$, if, for every time value $t$, we restrict avail $(S, t)$ to be $\operatorname{req}(S, E, t)$ then $E(t)$ is indeed computable.

It shall be clear that, in full generality, with arbitrary operators applied to streams, this is even not well-defined. Indeed, all $\operatorname{req}(S, E, t)$ for basic streams $S$ occuring in expression $E$ is presumably the solution of a set of interval equations that may not have least solution w.r.t. inclusion.

Our point is thus to restrict ouselves to some set of stream operators that guarantee the existence and computability of $r e q($,$) .$

\subsection{Delay operator}

\subsection{Reverse operator}

$$
\begin{gathered}
r e q(S, r l(S), t)=t \\
r e q(S, r r(S), t)=e(S)-t
\end{gathered}
$$




\subsection{Extract operator}

\section{Study Case}

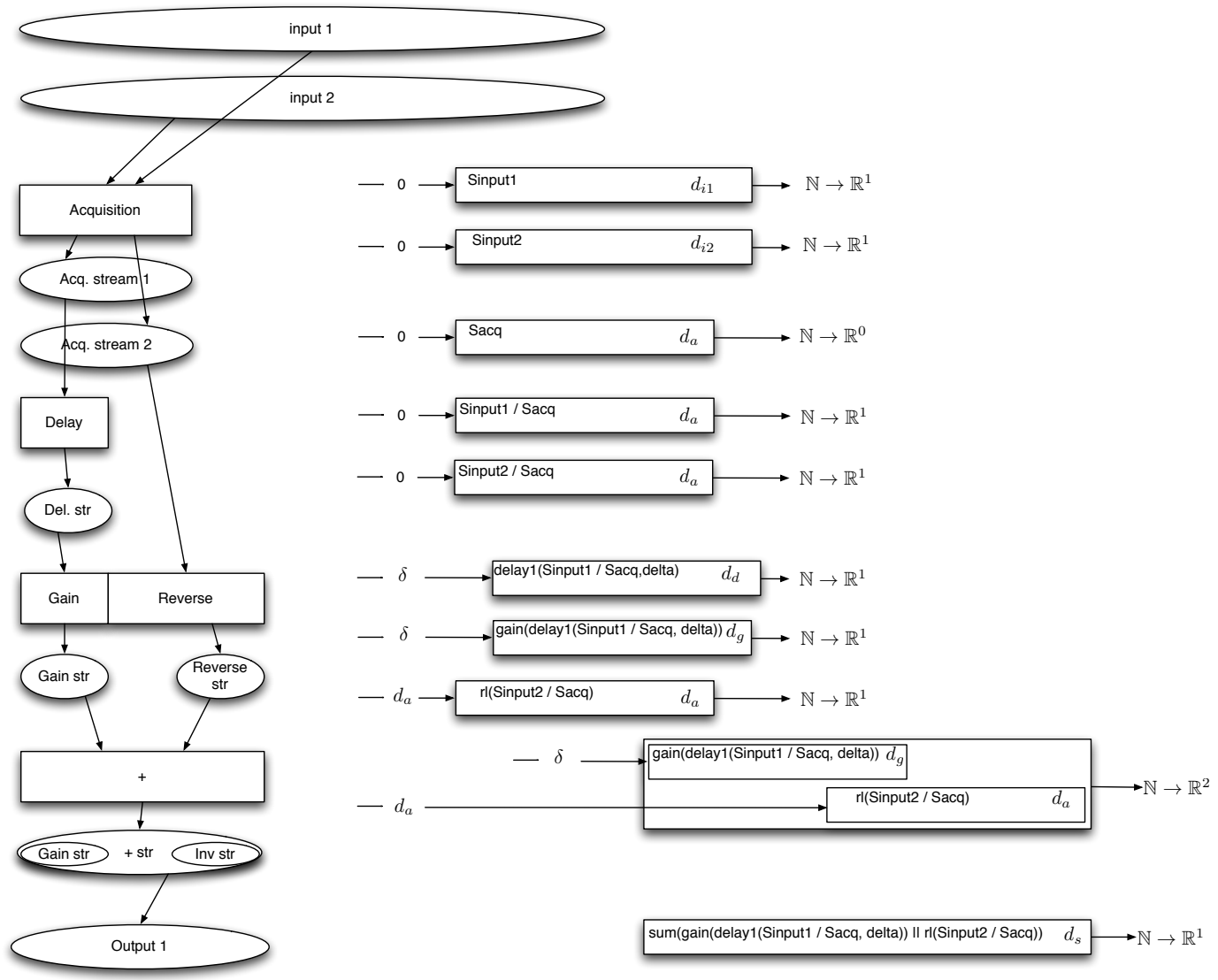

What are avail $\left(S_{\text {output } 1}, t\right), k\left(S_{\text {output } 1}, t\right), \operatorname{req}\left(S_{\text {output } 1}, t\right)$ ?

Process calculi has been applied to the modeling of interactive music systems $[18,17,23,28,16,27,1,24,19,4,14,11,13,15,2,22,12,20,21,10]$ and ecological systems $[25,6,26,7]$. In addition, research on algorithms $[5,3,9]$ and software engineering [8] also contributes to this field.

\section{And here we are for now... any comment wel- come}

\section{References}

[1] A. Allombert, M. Desainte-Catherine, and M. Toro. Modeling temporal constrains for a system of interactive score. In G. Assayag and C. Truchet, editors, Constraint Programming in Music, chapter 1, pages 1-23. Wiley, 2011. 
[2] J. Aranda, G. Assayag, C. Olarte, J. A. Pérez, C. Rueda, M. Toro, and F. D. Valencia. An overview of FORCES: an INRIA project on declarative formalisms for emergent systems. In P. M. Hill and D. S. Warren, editors, Logic Programming, 25th International Conference, ICLP 2009, Pasadena, CA, USA, July 14-17, 2009. Proceedings, volume 5649 of Lecture Notes in Computer Science, pages 509-513. Springer, 2009.

[3] J. D. A. Moreno, S. Passos, and M. Toro. On-line assembling mitochondrial DNA from de novo transcriptome. CoRR, abs/1706.02828, 2017.

[4] C. Olarte, C. Rueda, G. Sarria, M. Toro, and F. Valencia. Concurrent Constraints Models of Music Interaction. In G. Assayag and C. Truchet, editors, Constraint Programming in Music, chapter 6, pages 133-153. Wiley, Hoboken, NJ, USA., 2011.

[5] C. Patiño-Forero, M. Agudelo-Toro, and M. Toro. Planning system for deliveries in Medellín. ArXiv e-prints, Nov. 2016.

[6] A. Philippou and M. Toro. Process Ordering in a Process Calculus for Spatially-Explicit Ecological Models. In Proceedings of MOKMASD'13, LNCS 8368, pages 345-361. Springer, 2013.

[7] A. Philippou, M. Toro, and M. Antonaki. Simulation and Verification for a Process Calculus for Spatially-Explicit Ecological Models. Scientific Annals of Computer Science, 23(1):119-167, 2013.

[8] M. Raul, T. Mauricio, and C. Luz. Definicion de la arquitectura de referencia de un dominio: de la elucidacion al modelado. In R. Mazo, editor, Guia para la adopcion industrial de lineas de productos de software, pages 193-210. Editorial Eafit, 2018.

[9] J. M. C. Restrepo, A. F. Z. Palacio, and M. Toro. Assembling sequences of DNA using an on-line algorithm based on debruijn graphs. CoRR, abs/1705.05105, 2017.

[10] M. Toro. Exploring the possibilities and limitations of concurrent programming for multimedia interaction and graphical representations to solve musical csp's. Technical Report 2008-3, Ircam, Paris.(FRANCE), 2008 .

[11] M. Toro. Probabilistic Extension to the Factor Oracle Model for Music Improvisation. Master's thesis, Pontificia Universidad Javeriana Cali, Colombia, 2009.

[12] M. Toro. Towards a correct and efficient implementation of simulation and verification tools for probabilistic ntcc. Technical report, Pontificia Universidad Javeriana, May 2009. 
[13] M. Toro. Structured interactive musical scores. In M. V. Hermenegildo and T. Schaub, editors, Technical Communications of the 26th International Conference on Logic Programming, ICLP 2010, July 16-19, 2010, Edinburgh, Scotland, UK, volume 7 of LIPIcs, pages 300-302. Schloss Dagstuhl - Leibniz-Zentrum fuer Informatik, 2010.

[14] M. Toro. Structured Interactive Scores: From a simple structural description of a multimedia scenario to a real-time capable implementation with formal semantics. $\mathrm{PhD}$ thesis, Univeristé de Bordeaux 1, France, 2012.

[15] M. Toro. Structured interactive music scores. CoRR, abs/1508.05559, 2015.

[16] M. Toro. Probabilistic Extension to the Concurrent Constraint Factor Oracle Model for Music Improvisation. ArXiv e-prints, Feb. 2016.

[17] M. Toro. Probabilistic Extension to the Concurrent Constraint Factor Oracle Model for Music Improvisation . Inteligencia Artificial, 57(19):37-73, 2016.

[18] M. Toro. CURRENT TRENDS AND FUTURE RESEARCH DIRECTIONS FOR INTERACTIVE MUSIC. Journal of Theoretical and Applied Information Technology, 69(16):5569-5606, 2018.

[19] M. Toro, C. Agón, G. Assayag, and C. Rueda. Ntccrt: A concurrent constraint framework for real-time interaction. In Proc. of ICMC '09, Montreal, Canada, 2009.

[20] M. Toro and M. Desainte-Catherine. Concurrent constraint conditional branching interactive scores. In Proc. of SMC '10, Barcelona, Spain, 2010 .

[21] M. Toro, M. Desainte-Catherine, and P. Baltazar. A model for interactive scores with temporal constraints and conditional branching. In Proc. of Journées d'Informatique Musical (JIM) '10, May 2010.

[22] M. Toro, M. Desainte-Catherine, and J. Castet. An extension of interactive scores for multimedia scenarios with temporal relations for micro and macro controls. In Proc. of Sound and Music Computing (SMC) '12, Copenhagen, Denmark, July 2012.

[23] M. TORO, M. DESAINTE-CATHERINE, and J. CASTET. An extension of interactive scores for multimedia scenarios with temporal relations for micro and macro controls. European Journal of Scientific Research, 137(4):396-409, 2016. 
[24] M. Toro, M. Desainte-Catherine, and C. Rueda. Formal semantics for interactive music scores: a framework to design, specify properties and execute interactive scenarios. Journal of Mathematics and Music, $8(1): 93-112,2014$.

[25] M. Toro, A. Philippou, S. Arboleda, M. Puerta, and C. M. Vélez S. Mean-field semantics for a process calculus for spatially-explicit ecological models. In C. A. Muñoz and J. A. Pérez, editors, Proceedings of the Eleventh International Workshop on Developments in Computational Models, Cali, Colombia, October 28, 2015, volume 204 of Electronic Proceedings in Theoretical Computer Science, pages 79-94. Open Publishing Association, 2016.

[26] M. Toro, A. Philippou, C. Kassara, and S. Sfenthourakis. Synchronous parallel composition in a process calculus for ecological models. In G. Ciobanu and D. Méry, editors, Proceedings of the 11th International Colloquium on Theoretical Aspects of Computing - ICTAC 2014, Bucharest, Romania, September 17-19, volume 8687 of Lecture Notes in Computer Science, pages 424-441. Springer, 2014.

[27] M. TORO, C. RUEDA, C. AGÓN, and G. ASSAYAG. Ntccrt: A concurrent constraint framework for soft real-time music interaction. Journal of Theoretical \& Applied Information Technology, 82(1), 2015.

[28] M. TORO, C. RUEDA, C. AGÓN, and G. ASSAYAG. Gelisp: A framework to represent musical constraint satisfaction problems and search strategies. Journal of Theoretical \& Applied Information Technology, 86(2), 2016. 